\title{
Relaciones de equidad entre hombres y mujeres Análisis crítico del entorno familiar .
}

\author{
Isabel Vega-Robles \\ Instituto de Investigaciones Psicológicas \\ Universidad de Costa Rica \\ Dirección postal: 1283-2050, San José, Costa Rica \\ Ce: isabelv@racsa.co.cr
}

Resumen. Este artículo examina algunas concepciones y políticas relativas a la equidad de género en el ámbito familiar. Se considera que algunas de ellas con frecuencia excluyen dimensiones de problemas relacionados con el cambio social y los valores humanos. Con base en los aportes de varios estudiosos del tema, se señalan las limitaciones de los enfoques teóricos más utilizados para explicar la falta de equidad en las relaciones de género. También se toman los resultados de investigaciones sobre las opiniones y actitudes de la población costarricense, así como el contenido de alguna legislación relativa a la equidad de género y la violencia doméstica, para mostrar esa exclusión conceptual de dimensiones centrales de las relaciones de género. Esa limitación podría explicar la poca efectividad de las leyes para mejorar las relaciones familiares.

Palabras clave: relaciones de equidad, entorno familiar, relaciones de género, violencia doméstica.

\begin{abstract}
This paper questions some of the conceptions and policies relative to gender equality in the family scope. It considers that frequently these assumptions exclude fundamental dimensions of the problem in its relationship with social change and human values. Based on contributions from several studies on this subject, it shows the limitations of the theoretical approaches used to explain the lack of equality in gender relations. It also takes the results of studies of the Costa Rican values and attitudes, as well as the content of some legislation relative to domestic violence, emphasizing the conceptual exclusion of central dimensions of gender relations. This limitation could explain the ineffectiveness of repressive laws to improve the family relations.
\end{abstract}

Key Words: Gender equality, family environment, gender relationships, domestic violence.

\footnotetext{
${ }^{1}$ Una versión anterior de este trabajo fue presentada en el "Seminario Internacional Democracia de Género: una propuesta para mujeres y hombres del siglo XXI" realizado en San Salvador, El Salvador, el 5 y 6 diciembre de 2000, por iniciativa de la Fundación Género y Sociedad (GESO) y la Fundación Heinrich Böll (FHB).
} 


\section{Introducción}

Actualmente las mujeres hemos logrado avances importantes en los campos de la educación, el trabajo y la salud, aún cuando falte mucho por hacerse (CEPAL, 1994; Pautassi, Faur \& Gherardi, 2004). Sin embargo, el entorno familiar parece una trinchera que se resiste, de manera persistente, a dejarse penetrar por las ideas y aspiraciones de equidad entre hombres y mujeres.

Casi todos los éxitos en la obtención de un trato equitativo han sido obtenidos en el seno de la sociedad pero no dentro del matrimonio y la familia (Friedan, 1997). Muy pocas mujeres tienden a extender la equidad a la relación entre marido y mujer (Lockwood, 1984; Vega, 2001).

Esta situación, caracterizada por pocos logros en términos de equidad de género sobre todo en el espacio familiar, ha sido vista como un estancamiento y ha llevado a reflexionar acerca de las estrategias y vías para continuar avanzando hacia relaciones de género más equitativas.

En esa dirección, ha surgido la propuesta "democracia de género", al igual que otras como la engendered society en Estados Unidos o el nuevo contacto entre los géneros en España, la cual busca ampliar la discusión sobre los cambios en las relaciones de género y su impacto en las esferas social, económica y políticas actuales, así como dinamizar la agenda política a favor de la equidad de género para evitar su debilitamiento, postergación o incluso su aniquilamiento (Meentzen \& Gomáriz, 2003)

En esta discusión, sin embargo, hay dos cuestiones que se han constituido en los grandes ausentes en el análisis de la equidad de género. Estas son el cambio social y los valores humanos. En este artículo se lleva a cabo un repaso de las limitaciones que tienen, para algunas autoras, los enfoques teóricos utilizados hasta hace poco tiempo para explicar la falta de equidad en las relaciones de género, en contraste con otros enfoques recientes novedosos. Asimismo, se propone que existe una exclusión de aspectos socioculturales centrales en un análisis del problema en Costa Rica, para lo cual cabe tomar como punto de referencia empírica las opiniones y actitudes de la población costarricense sobre familia y género, así como el contenido de la legislación relativa a la equidad de género y la violencia doméstica. Igualmente, el tema de la violencia familiar, aparece para ilustrar como dicha carencia conceptual tiene mucho que ver con las explicaciones o los motivos de la poca efectividad de las leyes para atender problemas familiares -limitaciones reconocidas por las mismas feministas-, y cómo, al enfocar parcialmente el problema y recurrir a medidas casi siempre represivas, es probable que más bien estas últimas operen como obstáculos para avanzar hacia la equidad de género. 


\section{Cambio social y valores}

Preston (2000) nos dice que todas las sociedades están implicadas en un proceso de cambio social pero al ser este tan gradual, las personas que viven en sociedades muy tradicionales apenas están enterados de él. Las acciones de los individuos, de las organizaciones y de los movimientos sociales tienen impacto en la sociedad y pueden convertirse en catalizadores para el cambio social. No obstante, las acciones de los individuos se dan dentro del contexto de la cultura, de las instituciones y de poderosas estructuras heredadas del pasado, y generalmente, para que estos individuos efectúen un cambio social dramático, la sociedad en sí misma ha de ser un vehículo para el cambio. Existe una alta correlación entre el índice del cambio y las resistencias sociales y culturales a este. En épocas en que los miembros de una sociedad tienen la sensación de que el cambio está "fuera de control", el deseo por la continuidad se hace más extremo, dando como resultado idealizaciones del pasado.

El proceso de "cambio sociocultural" -siendo este un término más aceptado, al ser la sociedad y la cultura interdependientes (Preston, 2000)queda ampliamente reflejado en las transformaciones y tensiones que experimentan las familias en las sociedades occidentales, como parte de los cambios ocurridos con el ingreso de la mujer en el mercado de trabajo, el uso de anticonceptivos, las luchas feministas por la igualdad de oportunidades, y la importancia relativa del trabajo de cara a otros intereses vitales (Hakim, 2003; Vega, 2003).

En ese contexto, los esfuerzos por la equidad de género ${ }^{2}$ se concentran, entre otras, en dos cuestiones álgidas: el mercado laboral y la vida familiar. Eso se traduce en diversas elaboraciones teóricas en el mundo académico y acciones a nivel político, económico y jurídico, que buscan dar cuenta de los factores que pueden ayudar a identificar y explicar, en dichas esferas, los avances y estancamientos en términos de equidad Como se señaló anteriormente, lo que interesa aquí es enfatizar la importancia de incorporar la dimensión de los valores en el análisis de la equidad de género, y llamar la atención sobre el peso de ciertas concepciones estereotipadas en que se sustentan algunas leyes relativas a la dinámica familiar en la sociedad costarricense; detallar las distintas posiciones tanto teóricas como políticas al respecto, no es el objetivo de este trabajo. González (1996) ofrece una reseña de las principales corrientes teóricas feministas.

${ }^{2}$ Por equidad me refiero a la "igualdad de opciones e igualdad de capacidades para decidir y alcanzar la mayor y mejor expansión posible de realizaciones valiosas (vivir y vivir "bien"), como actores con dominio de nuestras vidas, haciendo que las diferencias sean atendidas con justicia, sobre la base de la proporcionalidad y la diversidad" (D’Elia, 2004, p. 5). Así, esta definición combina la igualdad con la diversidad entendiendo la equidad como "igualdad en las diferencias". En este trabajo aparece el término "igualdad" cuando es utilizado por alguno de los autores que se citan. 
Para Schwartz (2001) los valores son metas deseables y transituacionales, los cuales varían en importancia y sirven como principios en la vida de una persona o de otra entidad social. Los valores pueden motivar la acción, dándole dirección e intensidad emocional, operan como criterios para juzgar y justificar la acción y se adquieren, mediante la socialización, en los valores del grupo dominante y de la experiencia personal de aprendizaje. Aquello que diferencia a los valores, en cuanto a su contenido, es el tipo de meta motivacional que expresan: "los valores representan, en forma de metas conscientes, las respuestas que los individuos deben de dar a tres requisitos fundamentales: las necesidades de los individuos en tanto que organismos biológicos, los requisitos de la interacción social coordinada y los requisitos para el correcto funcionamiento y supervivencia de los grupos" (Schwartz, 2001, p. 56). De estos tres requisitos universales se derivan diez tipos motivacionales de valores: poder, logro, hedonismo, estimulación, autodirección, universalismo, benevolencia, tradición, conformidad y seguridad (ver Tabla 1).

Tabla 1

$V$ alores correspondientes a cada tipo motivacional

Los valores específicos que principalmente representan a cada tipo motivacional son:

Poder: poder social, riqueza, autoridad, reconocimiento social.

Logro: exitoso, ambicioso, capaz, influyente, autorrespeto.

Hedonismo: placer, disfrutar la vida.

Estimulación: atrevido, vida variada, vida excitante.

Auto dirección: creatividad, libertad, curioso, independiente, elegir las propias metas.

Universalismo: tolerancia, justicia social, igualdad, protección del ambiente.

Benevolencia: ayudar, honesto, no rencoroso, responsable.

Tradición: Respeto, compromiso y aceptación de las costumbres e ideas que proporciona la cultura tradicional o la religión, humilde, moderado.

Conformidad: Buenos modales, obediente, honra a padres y mayores, autodisciplinado

Seguridad: orden social, seguridad nacional, seguridad familia, limpio, sentido de pertenencia.

Así, los tipos motivacionales conforman una estructura de relaciones dinámicas que se puede identificar porque las acciones emprendidas para realizar cada tipo de valor, tiene consecuencias psicológicas, prácticas y 
sociales que pueden entrar en conflicto o ser compatibles con la realización de otro tipo de valores.

La teoría diferencia además entre tipos de valores pero plantea que, a un nivel más básico, los valores configuran un continuo de motivaciones relacionadas -por el énfasis motivacional compartido de los tipos valorativos adyacentes- dando lugar a una estructura circular. Los tipos de valores en oposición pueden resumirse concibiendo los valores organizados en dos dimensiones bipolares. Una dimensión contrapone los valores de apertura al cambio con los de conservación, oponiendo los valores autodirección y estimulación con los valores seguridad, conformidad y tradición. La segunda dimensión opone los valores de autopromoción con los de autotrascendencia, confrontando los valores de universalismo y benevolencia con los valores de poder y el logro (Schwartz, 2001).

\section{La evolución social en términos de equidad de género}

Los logros en el acceso a la educación y el aumento de la presencia femenina en el mercado laboral son transformaciones que se han ido gestando en las últimas décadas, con mayor o menor éxito en distintas sociedades. Algunos estudios realizados en los años ochenta dan cuenta de una transición hacia nuevas actitudes en lo que se refiere al trabajo femenino, los roles sexuales y la autoridad en el hogar, dando cabida a un cuestionamiento a las tendencias teóricas más utilizadas entonces para explicar esos comportamientos y actitudes.

Lo que se puede observar en los Estados Unidos, es un cambio de actitud hacia la aprobación de la participación de la mujer en el mercado laboral y que la mujer casada tenga un empleo fuera del hogar. Si bien en este último caso ha llegado a ser una opción aceptada, no es visto como una obligación y parece ser opcional y voluntario, aún cuando con frecuencia es económicamente necesario.

La división sexual de los roles no ha variado significativamente en lo concerniente a las labores domésticas, toma de decisiones e incluso, la socialización de niños y niñas en el hogar. Las esposas o compañeras tienen a su cargo la mayoría del trabajo doméstico y le dedican más tiempo que sus esposos.

La autoridad del esposo sostiene las relaciones conyugales de poder y a menudo no se toman en cuenta las necesidades y deseos de los demás integrantes del hogar. Los cambios se han dado más en los estilos de influencia que en la toma de decisiones u otros patrones de control. Es decir, se evidencian diferentes formas de poder y de estrategias de influencia según el sexo. La falta de autoridad tangible, concreta, lleva a la mujer a ejercer el 
poder a través de indirectas y encubiertas técnicas en las relaciones íntimas (Szinovacz. 1984). En un estudio sobre las tácticas de influencia en tres cohortes de hombres y mujeres puso de manifiesto que las mujeres tienden a utilizar estrategias indirectas/emocionales como por ejemplo el llanto, o el tratamiento silencioso, en las relaciones íntimas (Hoffman, 1982, citado por Szinovacz, 1984).

Para Szinovacz (1984), los enfoques teóricos utilizados hasta ese momento resultan limitados y parciales para instrumentar estrategias que promuevan una equidad de género. La teoría del intercambio supone que las contribuciones económicas del esposo son las bases de poder más importantes y más efectivas que las de la esposa, lo cual hace presumir que si la mujer tiene acceso a la economía y a otros valores sociales, su posición de poder con relación al esposo se incrementará. No obstante, las investigaciones transculturales no confirman que el trabajo de la mujer constituya una condición necesaria y suficiente para la equidad, con lo cual, es un supuesto a debatir.

Con respecto a lo anterior, es necesario tomar en cuenta que el mantenimiento de una relación de intercambio social dependerá de la relación entre las recompensas que se obtienen y los costes en que se incurre. Una conducta social tenderá a mantenerse cuando las recompensas son superiores a los costes (Álvaro \& Garrido, 2003).

En cuanto a las teorías o enfoques feministas, Lockwood (1984) considera que no han tomado conciencia de la complejidad de la relación entre la familia, otras estructuras sociales y la liberación femenina. En consecuencia sus sugerencias para explicar el cambio familiar son inadecuadas, ya sea desde el enfoque marxista, con un énfasis en la condición de sumisión y explotación; la tendencia centrada en la naturaleza patriarcal de la sociedad; o bien, la que integra ambas y plantea como solución una transformación tanto de la estructura social como de la familia.

Esta autora sugiere que los movimientos feministas de los siglos XIX y XX no han traído cambios significativos en los patrones familiares debido a la complejidad teórica de las relaciones entre la familia, la estructura social y el estatus de la mujer; los problemas prácticos del establecimiento de relaciones equitativas en el hogar, aún si las esposas logran equidad en el mundo del trabajo, y al alto valor de la maternidad en la sociedad, muy extendido y en aumento en la mujer, sea o no feminista.

Szinovacz (1984) y Lockwood (1984) comparten el criterio según el cual el peso de la maternidad es determinante en todo esto: como valor, como práctica social y tal vez por las características biológicas y psicosociales del vínculo madre-niño/a. La maternidad no sólo afecta el potencial competitivo de la mujer en el mercado laboral, también la hace más dependiente que al hombre en el mantenimiento de unas relaciones íntimas estables y relativamente seguras. La aplicación unilateral de los estándares de 
autonomía por parte de los hombres es probablemente reforzada por la creciente demanda de autonomía de las mujeres, resultando en conflictos y luchas de poder en las relaciones íntimas (Szinovacz, 1984).

Estos estudios hacen evidente, cómo los valores sociales, familiares y de género, definen múltiples necesidades e intereses en constante confrontación. Sin embargo, este aspecto medular había sido escasamente analizado hasta hace muy poco tiempo, cuando surgen aproximaciones teóricas alternativas.

Hakim (2005) nos brinda una explicación novedosa -alejada de prejuicios y con datos empíricos fiables- acerca de las orientaciones hacia el empleo y los roles familiares en España y Gran Bretaña, países muy representativos de los procesos sociales que están ocurriendo en las sociedades modernas opulentas. Asumiendo que estas últimas ofrecen a la gente una amplia variedad de estilos de vida entre los cuales elegir, analiza los modelos de familia ideal y las preferencias asociadas sobre roles sexuales y orientaciones laborales.

Para Hakim (2005), la Comisión Europea impulsa sólo el modelo de familia sueco, en donde todos las adultos sanos trabajan de por vida y los servicios públicos se ocupan del cuidado de los hijos. Sin embargo, este modelo no es el preferido en Europa occidental. Por el contrario, en Europa occidental y Norteamérica se respaldan tres tipos distintos de familia: el de compromiso, el cual requiere cierto grado de especialización de rol en la familia, en donde por lo general es la mujer quien asume la responsabilidad principal del trabajo doméstico y familiar (Alemania occidental, Luxemburgo e Irlanda); el igualitario, con una división igualitaria del trabajo remunerado y familiar (Dinamarca, España y Gran Bretaña); y el de roles separados, cuando sólo el marido trabaja y la mujer se ocupa de la casa. No hay una división norte-sur y el modelo de familia no está determinado por la prosperidad económica.

La gran mayoría de las mujeres europeas no adopta nunca la identidad del (co)sustentador primario implicada en unos roles familiares genuinamente simétricos (excepto en Dinamarca, pero sólo la mitad). En los años ochenta, en la mayoría de los países sólo una minoría acepta esa responsabilidad, en una proporción que va de un quinto a un tercio. España tiene la menor proporción de mujeres que se consideran sustentadoras primarias, solo un 18\%. Esa realidad contrasta con el idealismo expresado por los españoles en las encuesta del Eurobarómetro, en una proporción de $50 \%$ de adultos que apoyan el modelo igualitario (Hakim, 2005).

El panorama no cambió en los años noventa ni en EEUU ni en Europa. El "International Social Survey Programme" ISSP muestra que la opinión estaba igualmente dividida en la respuesta respecto a la idea de que "el cometido del marido es ganar dinero y el de la mujer cuidar de la casa y la familia", a pesar del creciente apoyo público al derecho de la mujer a 
participar en el mercado laboral (Scott et al., 1996, citado por Hakim, 2005, p. 5).

Hasta hace muy poco tiempo no ha habido teorías generales (Parsons \& Bales, 1955; Becker, 1981, 1991; Walby, 1990, citados por Hakim, 2005) que predijesen la marcha de las orientaciones laborales y la división familiar del trabajo en las sociedades modernas ricas que no fueran rápidamente invalidadas por la evidencia. Todas esas teorías comparten el supuesto de la homogeneidad en las aspiraciones y las preferencias de las mujeres y los hombres, en los modelos de familia ideal y en las historias de empleo.

El estudio en cuestión parte de la teoría de la preferencia, que plantea al menos tres modelos ideales de familia sustancialmente diferentes -el igualitario, el de compromiso y el de segregación de roles sexuales- y preferencias heterogéneas de las mujeres por una vida centrada en una carrera pública, una vida centrada en el hogar y la familia o cierta combinación de las dos (Hakim, 1999 a, 1999 b, 2000 a, 2001 b, 2002; citado por Hakim, 2005).

Hakim (2005) encontró que ahora en Gran Bretaña hay un rechazo en la mayoría, a una segregación rígida de roles en la familia, así como a la ideología de los roles sexuales que asignan el rol de sustentador exclusivamente a los hombres y el rol de ama de casa exclusivamente a las mujeres. Sin embargo esa situación no ha implicado la aceptación de un nuevo modelo de roles sexuales totalmente simétrico, en el cual el hombre como la mujer son responsables, en el día a día, de los ingresos y del trabajo doméstico. Más bien, la frontera entre los dos roles se ha difuminado y una mayoría acepta que cada miembro puede y debe sustituir al otro, in extremis, así como acepta la división sexual del trabajo en la familia con un alto grado de flexibilidad en el mundo moderno.

Con relación al compromiso con el trabajo, en la vida de la mujer, el empleo y otras actividades de la esfera pública son cada vez más importantes, mientras que, por el contrario, los hombres creen que ahora es socialmente aceptable que ellos tengan otros intereses vitales al margen de su empleo. Ahora bien, el compromiso con el trabajo ha seguido influido por la ideología femenina de los roles sexuales, y así, el compromiso con el trabajo disminuye a niveles mínimos entre las mujeres inactivas, quienes prefieren el modelo de familia de roles segregados y creen que el papel de sustentador ha de ser siempre del hombre.

En España, una mayoría -tres cuartas partes de hombres y mujeres- cree que ambos miembros de la pareja deben compartir la responsabilidad de aportar ingresos y trabajo doméstico in extremis. Solo una minoría, un 38\%, está de acuerdo en que incluso cuando la mujer tiene trabajo, el hombre sigue siendo el sustentador principal, cifra por debajo del $56 \%$ que se observa en Gran Bretaña. Sin embargo, entre las parejas cohabitantes o casadas de 18 años o más, cuatro quintos de los esposos y esposas dijeron 
que el marido era el sustentador principal, unas proporciones ligeramente mayores que en Gran Bretaña (Hakim, 2005, p. 75)

Los resultados de una encuesta en España muestran un considerable sesgo de corrección política (deseabilidad social) ${ }^{3}$. Sin embargo los españoles son aún más proclives que los británicos a manifestar que prefieren el modelo familiar de roles simétricos. La autora concluye:

"la adopción de la identidad y las responsabilidades del sustentador primario no vienen determinadas principalmente por la ocupación y nivel de ingresos, como tan frecuentemente han dicho las feministas. (...) La percepción que tienen las personas de sí mismas como sustentadores principales (conjuntas) de su familia, como sustentadoras secundarias o como dependientes está moldeada por su ideología de los roles sexuales más que por su estatus laboral" (Hakim, 2005, p. 148).

El estudio de Hakim (2005) permite constatar el peso de la diversidad de modelos ideales de familia, de las preferencias de estilos de vida tanto en lo laboral como en lo doméstico y de la ideología de género, al intentar explicar el comportamiento del mercado de trabajo y de los roles sexuales en la familia. Esa manera de abordar el tema en cuestión -los avatares de la equidad de género- permite confrontar las ideas y los valores socialmente legitimados con las prácticas cotidianas.

Una investigación de Björnberg y Kollind (2003) apunta en esa misma dirección, y muestra la importancia del significado de la responsabilidad conjunta en la distribución del trabajo doméstico. Para estas autoras, lo usual ha sido buscar similitudes y diferencias en la cantidad de tiempo y de trabajo que hombres y mujeres invierten en él, es decir, la igualdad se define en términos de semejanza o de lo idéntico. Distanciándose de ese enfoque, ellas se interesaron por comprender la manera en que las personas entienden la equidad de género en la vida familiar, sin imponer nociones definidas a priori (sociología de comprensión o entendimiento).

Mediante entrevistas a profundidad realizadas con veintidós parejas suecas, buscaron conocer algunos de los principios o reglas a las que recurren hombres y mujeres cuando intentan explicar o justificar el modo como organizan el trabajo doméstico en sus economías, el significado de la igualdad y qué encuentran justo o injusto en sus vidas domésticas. Una de las conclusiones del estudio resalta cómo para las parejas entrevistadas -mujeres

\footnotetext{
${ }^{3}$ Los científicos sociales españoles señalan que la "igualdad" es la nueva ideología moderna de la posdemocracia que apoya todo el mundo en España. Sin embargo, las actitudes y valores igualitarios apenas guardan relación con el verdadero comportamiento de los españoles (Hakim, 2005, p.77).
} 
y hombres por separado- asumir la plena responsabilidad no quiere decir que tengan que hacer las mismas tareas o dedicarle la misma cantidad de tiempo a las tareas domésticas. Al expresarse sobre la responsabilidad conjunta "los entrevistados no subrayaron en ningún momento la dimensión de similitud" (Björnberg \& Kollind, 2003, p. 159). En lo que insistieron fue en que ambos debían ver lo que hay que hacer y hacerse responsables de lo que es de interés común.

Este estudio da cuenta de la dimensión subjetiva de hombres y mujeres, quienes, desde un sistema de valores culturales compartido, otorgan sentido a sus prácticas cotidianas (Berger \& Luckmann, 1984). De esa forma, emerge el espacio de tensión entre lo macro y lo microsocial en el cual podemos hallar algunas pistas que nos ayuden a comprender el aparente "sin sentido" de muchos de sus comportamientos.

Hakim (2005) y Björnberg y Kollind (2003) trascienden las limitaciones de algunas concepciones teóricas feministas sobre las relaciones de equidad de género -con un énfasis en factores económicos, educativos o discriminatorios para las mujeres- y muestran la estrecha vinculación existente entre las actitudes y los valores de hombres y mujeres y las distintas pautas de comportamiento en lo laboral y en la vida familiar, en el contexto de la sociedades desarrolladas.

A continuación, guardando las distancias que han de prevalecer cuando hacemos referencia a comportamientos observados en otros países y culturas, veremos como Costa Rica muestra ciertas similitudes con algunas de las tendencias observadas en las sociedades desarrolladas.

\section{Trabajo, vida familiar y entorno social: el caso de Costa Rica}

En las últimas dos décadas, las opiniones y comportamientos de la población costarricense sobre diversos aspectos de la vida cotidiana dejan traslucir una amalgama de percepciones -a menudo aparentemente incongruentes- sobre el grado de felicidad, lo precario de la situación económica y política de la sociedad en general y las expectativas acerca de las relaciones familiares.

En los años ochenta, las mujeres costarricenses de escasos recursos manifiestan que trabajan debido a la urgencia de aumentar el ingreso familiar y su participación varía de acuerdo al nacimiento y cuidado de los hijos. En los estratos medios el trabajo de las mujeres se asocia a deseos de realización personal, independencia económica o de aportar al presupuesto familiar, pero la autoridad del hogar se deposita en manos del esposo, a quién se le 
atribuye la última palabra en la toma de decisiones (Barrantes, et al., 1979; citado por Vega, 1993).

En los años noventa, esas ideas tienden a mantenerse y los valores que subyacen en las opiniones y actitudes de la población, son compartidos tanto por hombres como por mujeres. Las ideas de autonomía y equidad propias de la modernidad son percibidas como una amenaza a la felicidad y armonía familiares. Esta y otras creencias de corte tradicional se ven reflejadas en la forma como se organizan las familias para cubrir las necesidades -materiales y afectivas- de todos sus integrantes.

De la información aportada por múltiples estudios y encuestas de opinión (Herrera, 1999; Méndez, 1999; Gólcher, 2000; Instituto de Investigaciones Psicológicas UCR \& Organización Panamericana de la Salud, 1996; Centro Mujer y Familia, 1997), podemos observar que la familia es lo más importante en la vida de los y las costarricenses, y que le otorgan altos grados de importancia al trabajo, tiempo libre, religión, deportes y amigos, en ese orden. Para la juventud, sus principales metas y aspiraciones son tener una familia, ser felices, lograr éxito en la vida y tener una buena relación, en paz, con la familia. Sin embargo, en general la gente se muestra crítica, pesimista, insatisfecha y desconfiada en lo que respecta a la política, al papel de las instituciones y a las condiciones económicas y de seguridad en su comunidad y en el contexto nacional.

Acerca del futuro, una significativa proporción vaticina que en el futuro habrá igualdad total en la relación de pareja, el trabajo del hogar se repartirá en forma equitativa entre hombres y mujeres y la educación de los hijos será una responsabilidad de ambos padres por igual. Paradójicamente, el hombre seguirá siendo machista, percepción más frecuente entre las mujeres que entre los hombres y las opiniones acerca de si la mujer podrá hacer todo lo que hace el hombre se distribuye por partes iguales entre quienes consideran que así será y quienes creen lo contrario. No obstante, si bien casi la totalidad de la población considera que una mujer no sólo se realiza como madre y esposa sino también como profesional, más de la mitad estima que cuando la mujer trabaja, por más que trate de cumplir, siempre abandona su casa y todo camina mal. La mitad de la población afirma que si una mujer participa en política, el hogar se perjudica con su ausencia. Si una mujer gana más que su marido es casi seguro que eso traerá problemas y ser ama de casa es tan satisfactorio como trabajar a sueldo o salario y existe desacuerdo en que una madre que trabaja puede tener una relación tan cálida y segura con sus hijos como una que no trabaja. Además, un niño necesita tener un hogar con un padre y una madre para ser feliz ${ }^{4}$. El matrimonio no es considerado una

${ }^{4}$ En Costa Rica, sólo un poco más de la mitad de los hogares están formados por la pareja conyugal e hijos y alrededor de una décima parte por uno solo de los padres y sus hijos, en su gran mayoría mujeres. Del total de hogares, casi la quinta parte de las jefaturas del hogar está a cargo de mujeres (69.690 mujeres) (Herrera, 1999; Vega, 1993). 
institución pasada de moda y se cree que el propio matrimonio o unión se mantendrá estable. La mayor aspiración es contar con una casa propia y un matrimonio feliz (Dobles \& Ruiz, 1996).

Existe temor a las implicaciones que puede tener en el ámbito familiar la participación de las mujeres en el mundo del trabajo y la política, aprehensión más frecuente entre las mujeres que entre los hombres. Asimismo, ellas, con mayor frecuencia que los hombres, comparten la convicción de que la responsabilidad total de mantener el hogar corresponde a ellos. Es más, cuando las mujeres viven en pareja, su opinión favorable a la jefatura masculina es mayor que la de los hombres con pareja, aún cuando la mujer con más instrucción está menos dispuesta a la jefatura masculina y los hombres con educación superior se inclinan más que las mujeres universitarias, a una jefatura compartida (CMF, 1997).

Considerando los resultados de estas encuestas podemos decir, que la sociedad costarricense es tradicional, benevolente y conformista, con valores conservadores que se contraponen a aquellos de auto-dirección y logro, y por ende, a una apertura al cambio (Schwartz, 2001). Predomina la moderación y una inclinación a que los cambios sean graduales, porque, según la mayoría, traen problemas y sufrimiento para otras personas, aunque hay quienes opinan -los de mayor educación- que los mismos traen cosas buenas y progreso (Gómez, 1996) .

Igualmente, esos estudios ponen de manifiesto como las propias mujeres aparecen ambivalentes con respecto a una innovación sustantiva hacia relaciones de género más equitativas, posiblemente porque un cambio en ese sentido implica también ceder espacios y funciones en ámbitos que, como el hogar y la maternidad, constituyen un eje fundamental de su identidad y de ciertas formas de poder. La familia es una institución en la cual la mujer construye, lidera, organiza, practica y reproduce buena parte de las reglas del juego que queremos cambiar (Vega, 2000; Limone, 2003).

Vega (2000) analizó la relación familia-trabajo en un estudio cualitativo con once mujeres microempresarias y mostró que la mujer, como agente generador y sostenedor de vida, influye de forma determinante en la dinámica interaccional de la familia. Para Limone (2003), el machismo no sólo lo evidencian los varones (y no todos ellos) sino también algunas mujeres, que socializadas en un sistema patriarcal, participan de la creencia en la división tradicional de roles sexuales y la reproducen en su quehacer diario como trabajadoras asalariadas, como madres y en su discurso.

Así pues, podemos esperar pocos resultados de las propuestas y acciones sociales concebidas para favorecer el cambio, si no se presta la atención debida al papel que juegan los valores sociales en el

\footnotetext{
${ }^{5}$ Una descripción más detallada de los estudios resumidos aquí se puede encontrar en Vega (2001).
} 
comportamiento de cada una de las partes -hombres y mujeres- involucradas en ese proceso ${ }^{6}$.

\section{Cambio de perspectiva en la búsqueda de equidad de género}

Los pocos logros en cuanto a equidad de género en el entorno familiar nos conducen a una reflexión sobre la importancia de un cambio de perspectiva teórica que incluya la dimensión de los valores y actitudes.

El repaso de las investigaciones recientes da cuenta de un viraje hacia nuevas concepciones del problema, en donde los valores adquieren suma importancia en el comportamiento del mercado de trabajo y el desempeño de hombres y mujeres en el seno de las familias, entre otros temas.

En Costa Rica la legislación para atender problemas de las familias se sustenta en ideas estereotipadas sobre sus causas y posibles soluciones, y la vinculación de las luchas feministas con el desarrollo de conocimiento y de leyes sobre esos tópicos, por ejemplo la violencia intrafamiliar, es evidente.

Un análisis de la CEPAL (1993) nos dice que en Latinoamérica los estudios se han orientado exclusivamente a describir las condiciones de las mujeres -usualmente esposas y madres- en la evolución de las estructuras y el funcionamiento de las familias. Sin embargo, el hecho de que las transformaciones socioeconómicas han incidido dramáticamente en las mujeres de los sectores populares, no justifica que los estudios sobre la familia sigan centrados en la situación de la mujer. "Los esfuerzos de investigación y de búsqueda de antecedentes para la formulación de políticas deberían estar orientados más bien a aumentar nuestra comprensión de los procesos de desorganización familiar que conducen a esos resultados" (CEPAL, 1993, p. 112).

Volcando la mirada hacia nuevas interrogantes sobre el lugar de los hombres en esta coyuntura social, ese mismo organismo latinoamericano considera que diversos factores como la alteración en las aspiraciones y expectativas de los estilos de vida de las personas, la inercia de los patrones culturales tradicionales en donde la división del trabajo familiar designa al hombre como proveedor único, los avances educativos, una disminución de la fecundidad y por consiguiente, la posibilidad de que ambos cónyuges se inserten en el mercado de trabajo, condujeron a un complejo entramado de causas y efectos que transformaron la posición de los hombres en las familias. Como parte de este proceso, se da un distanciamiento entre los roles tradicionales y el acceso legítimo a su desempeño, lo que lleva a "el

${ }^{6}$ Cuadrado, Navas y Molero (2003) encontraron cierta ambigüedad en los cambios respecto a la estereotipia de roles. Si bien se observa un cambio en la valoración del trabajo doméstico, este se mantiene asociado a la feminidad, aspecto sobre el que no han incidido las políticas de igualdad y constituyen una barrera para la democracia de género. 
cuestionamiento a la distribución del poder intrafamiliar y el debilitamiento de la autoestima de los hombres" (CEPAL, 1993, p. 114) situación que deriva en una pérdida de confianza en la propia capacidad para cumplir con sus responsabilidades de esposo y padre "actitudes que probablemente constituyen el trasfondo del comportamiento 'irresponsable' de los hombres pertenecientes a los sectores populares urbanos" (CEPAL, 1993, p. 114). Ese desgaste de las bases de la autoridad del padre puede inducir a la violencia familiar, como un intento de este último de buscar imponer su voluntad sobre el resto de los integrantes de la familia de manera autoritaria (De Oliveira \& García, 1991, citado por CEPAL, 1993, p. 116).

Para Gil, Pujal, Nañez, Laira y Martínez (2003) la aparición de nuevas y diferentes agrupaciones familiares, coexiste simultáneamente a la persistencia del imaginario de familia nuclear y la violencia doméstica denota esa fractura de la familia nuclear:

"La excesiva presión a la que están sometidos muchos varones a partir de la precarización y flexibilidad laboral afecta tanto a la producción de su existencia como a su subjetividad en términos simbólicos (dada la pérdida de referente identitario), más la amenaza de abandono -imaginada o realpara muchos varones por parte de muchas mujeres (...) han hecho emerger de manera virulenta el último y desesperado intento que le queda al varón para sentirse importante y no perder todo punto de referencia identitario" (p. 236).

\section{Legislación a favor de las mujeres: ¿logro o estancamiento?}

Costa Rica resulta un ejemplo paradigmático, con índices de desarrollo en salud y educación propios de países desarrollados, pero con grandes aprensiones hacia un cambio en las relaciones de género.

Los medios de comunicación y las voces políticas -gobierno, instituciones públicas y privadas- denuncian desigualdades y promueven una legislación a favor de las mujeres que, como vimos, no coinciden con muchas de las concepciones y prácticas de una mayoría, dándose una distancia a veces abismal entre ambas esferas de la realidad social costarricense.

Por otra parte, las luchas a favor de las mujeres -llevadas a cabo por distintos colectivos feministas e instituciones sociales- se basan sobre todo en la denuncia de situaciones de injusticia y desigualdad y de un gran activismo en la promoción de leyes para combatirlas. 
Estas acciones parecen sustentarse en la idea de que un cambio hacia la equidad de género será posible si, por una parte, se denuncia el abuso de poder a que se han visto sometidas las mujeres en el ámbito laboral, político y familiar y, por otra, si se legisla a favor del colectivo femenino para lograr su protección y bienestar. En este sentido, se han denunciado discriminaciones hacia la mujer en el mundo del trabajo, el hogar y el sistema de salud y bienestar social en general, obteniendo éxito en la promulgación de un cuerpo de leyes a favor de las mujeres.

De esa manera, la producción de leyes, decretos y programas a favor de la equidad de género se ha dirigido principalmente hacia la mujer y su situación en el entorno familiar, en un esfuerzo por atender problemas como la violencia o el hostigamiento sexual principalmente, aunque también se han tratado situaciones como las niñas o adolescentes embarazadas, la maternidad, la lactancia materna, las relaciones de hecho y las pensiones alimenticias (CMF, 1998).

La Ratificación de la Convención Interamericana para Prevenir, Sancionar y Erradicar la Violencia contra la Mujer y la Ley Contra el Hostigamiento Sexual en el Empleo y la Docencia son legislaciones que circunscriben el problema de la violencia a sus efectos sobre el colectivo femenino, actuada por los hombres hacia la mujer o entre ellos. Las estadísticas sobre agresión física respaldan con creces este propósito, pero en sus planteamientos se obvia el problema de la violencia psicológica actuada también muy frecuentemente por las mujeres, la cual ha sido encontrada en.un estudio sobre la incidencia de hechos de violencia realizado en 1996 mostró que los gritos y la descalificación hacia el cónyuge son conductas frecuentes tanto en hombres como en mujeres (Dobles \& Ruiz, 1996).

$\mathrm{Al}$ no tomarse en cuenta los distintos planos del problema ni a todos sus protagonistas, los anteriores resultan instrumentos de utilidad muy limitada para erradicar la violencia en las relaciones interpersonales y sociales.

A su vez, la Ley Contra la Violencia Doméstica, asentada en el principio de igualdad social, está dirigida a la familia pero su motivación básica es la protección de la mujer. Esta ley, aprobada en abril de 1996, otorgó a las víctimas de agresión posibilidades de solicitar medidas de protección y permitió acciones inmediatas por parte de los Juzgados de Familia y la Fuerza Pública.

Sin embargo, su principal ventaja, cual es la de permitir el trámite expedito, es también objeto de fuertes críticas. Los supuestos agresores, en su mayoría hombres, se quejan de que les violenta sus derechos. Y es que la ley obliga a los jueces a dar el beneficio de la duda a la mujer y girar inmediatamente una medida de protección sin tener pruebas contra el presunto agresor. Con esa orden, la Fuerza Pública debe notificar al agresor y si es necesario, sacarlo de su domicilio. Si no se respeta la orden se expone a una acusación por desobediencia a las autoridades. Uno o dos meses después 
viene la audiencia en donde ambas partes dan su versión y queda a criterio del juez decidir si confirma o no la medida. Si falta alguna o ambas partes a la audiencia, la medida de protección quedará sin efecto.

Para algunas autoridades, el hecho de que las mujeres no asistan a la audiencia puede deberse a que el presunto agresor entra en proceso de reconocimiento sensibilidad y reconciliación. La mujer confía en que él va a cambiar ya sea por un vínculo de pareja, por los hijos, por dependencia económica o psicológica (Vizcaíno, 1999).

Nuevamente, se hacen evidentes las omisiones e inconsistencias del discurso oficial, pues si bien en esa declaración se reconoce el papel de la mujer como agente activo en la cadena de la violencia, ocurre lo contrario a la hora de plantear soluciones.

$\mathrm{Y}$ es que al no cumplir la citada ley las expectativas iniciales, se ha propuesto otra ley aún más drástica para penalizar la violencia contra las mujeres mayores de edad.

La Comisión Especial de la Mujer de la Asamblea Legislativa dictaminó por consenso, el 22 de agosto del 2000, el proyecto de Ley de Penalización de la violencia contra las mujeres mayores de edad. Esta ley contempla duras sanciones para la violencia física, psicológica, sexual y patrimonial contra las mayores de 18 años. Establece un nuevo tipo penal denominado "femicidio" que sanciona con prisión de 20 a 35 años a quien mate a una mujer, siempre que mantenga o haya tenido una relación de poder o confianza: vínculos de convivencia, afectivos de jerarquía o autoridad. La propuesta se sumaría a la protección existente en la Ley contra la violencia doméstica, Ley integral para la persona adulta mayor y Código de la Niñez y la Adolescencia ${ }^{7}$.

Además, no admite la conciliación en los delitos de carácter sexual en prejuicio de menores o por violencia contra las mujeres. El proyecto también propone otras novedades como las siguientes: prisión (de 3 meses a 3 años) al funcionario que obstaculice el acceso a la justicia o bien por la omisión de funciones cuando esté en riesgo la vida e integridad de una mujer; cárcel (8 meses a 3 años) por la explotación económica de la mujer; y se incrementa la pena en casos de disminución de la calidad de vida, empobrecimiento, limitación de su desarrollo profesional, laboral, deportivo o artístico, impida o prohíba el uso, disfrute o enajenación de los bienes patrimoniales de la mujer; se agrava el castigo en casos de violencia sobre la persona, intimidación o amenazas contra ella y sus descendientes (Méndez, 2000).

Las reacciones a esta ley no se hicieron esperar y surgió la propuesta de un nuevo texto para penalizar la violencia doméstica, fundamentada en una reforma a varios artículos del Código Penal. La iniciativa ha pretendido sustituir el proyecto de Ley de Penalización de la violencia contra las mujeres mayores de edad. En la exposición de motivos de este proyecto se deja claro 
que la intención es la de evitar la duplicidad con respecto a la legislación penal actual. Además, se busca abrir el ámbito de aplicación de la normativa no sólo a mujeres mayores de edad, sino también a hombres, niños y ancianos (Venegas, 2000).

Ahora bien, si como muestran las encuestas en Costa Rica, la vida familiar se desenvuelve orientada por actitudes y valores que comparten hombres y mujeres, ¿porqué culpabilizar de lo que acontece a una de las partes? ¿Qué sentido tiene aplicar la fuerza y las medidas represivas para hacer entrar en razón a un colectivo que cree que hacer las cosas de otra manera puede tener nefastas consecuencias? Como lo manifestó un investigador en un reportaje de la prensa escrita: "la capacitación para cambiar los patrones machistas no sólo debe dirigirse a los varones; las mujeres también lo necesitan" (Gólcher, 2000).

Las medidas represivas deben existir como recursos extremos para atender situaciones de vida o muerte, pero si no se cambia la perspectiva con la cual se analiza el problema, poco podremos hacer para prevenir su gestación.

\section{¿Nuevos caminos hacia la equidad de género?}

Como sabemos, la identidad de género y las pautas de interacción hombre-mujer se construyen recíprocamente a través del proceso de socialización en la familia y otras instituciones sociales. La institución familiar regula funciones esenciales para la sobrevivencia humana, sustentada en un sistema de valores socialmente compartido.

El enfoque feminista no ha prestado la debida atención a este principio básico. Ha obviado que los aspectos de la cultura tempranamente aprendidos son resistentes al cambio, por el gran esfuerzo requerido para cambiar elementos centrales de la organización cognitiva de un adulto y porque los valores más básicos que se poseen tienden a ser afines en sí mismos y su abandono produciría una gran incertidumbre y ansiedad (Inglehart, 1991). El problema es que el cambio social acelerado supera lo conocido y surgen la resistencia, la tensión y el conflicto.

En las condiciones económicas, políticas y económicas actuales, la conquista del universo productivo y el ejercicio del poder político han sido las metas fundamentales de las luchas feministas, sin ahondar en una valoración sustantiva del peso que tiene la familia tradicional como construcción simbólica y fundamental en nuestra sociedad. La amenaza que representa el cambio y las resistencias que suscita en los y las protagonistas de la trama, son interpretadas como actos de dominio masculino, intencionalmente ejercidos sobre mujeres pasivas que se mueven en un universo cuyos valores les son ajenos. 
De esta manera, se reconoce el poder manifiesto de los hombres, sin una perspectiva de proceso que dé cuenta del estilo y cuotas de poder de hombres y mujeres en los distintos ámbitos y dimensiones de la realidad social y familiar.

Como de una u otra manera lo muestran los estudios citados previamente, ese modelo unidimensional y causal para analizar las relaciones de género, deja por fuera la tensión entre los distintos planos -laboral y familiar- de la vida cotidiana, la tirantez entre las expectativas individuales y los valores familiares, y la complejidad de las diferentes estrategias y formas de control que ejercen hombres y mujeres.

Paradójicamente, las medidas de corte autoritario y represivo, tan frecuentemente asociadas al comportamiento masculino, son las más recurridas a la hora de promocionar leyes a favor de las mujeres. Al no lograrse el resultado deseado, se tiende a promover leyes aún más represivas, como la Ley de penalización de la violencia contra mujeres mayores de edad (2007).

Así pues, el tema de la equidad de género debe abordarse desde la multiplicidad de factores y la variedad de sus manifestaciones, considerando que el fenómeno tiene que ver con los cambios sociales, los valores y los modelos de socialización. Es fundamental propiciar una crítica constructiva mediante el desarrollo de programas educativos formadores de pensamiento crítico y autoreflexivo.

\section{Referencias}

Álvaro J. L. \& Garrido, A. (2003). Psicología social. Perspectivas psicológicas y sociológicas. Madrid, España: McGraw Hill/Interamericana de España.

Berger P. \& Luckmann, T. (1984). La construcción social de la realidad. Argentina: Amorrortu.

Björnberg, U.; Kollind, A.K. (2003). Discurso sobre igualdad de género en dos familias asalariadas .Exploración de aspectos multidimensionales. En A. Meentzen \& E. Gomáriz (Eds.). Democracia de género. Una propuesta inclusiva (pp.151-180). El Salvador: Fundación Heinrich Böll.

Centro Mujer y Familia (CMF) (1997)). Encuesta Nacional: masculinidad, salud reproductiva y paternidad responsable. (Informe preliminar). San José, Costa Rica.

Centro Mujer y Familia (CMF) (1998). Avances legales hacia la equidad de género: leyes, proyectos de ley y decretos ejecutivos sobre derechos de las mujeres, equidad de género y familia: período 1994-1998. Centro Mujer y Familia/ Asamblea Legislativa. San José, Costa Rica.

Comisión Económica para América Latina (CEPAL) (1993). Cambios en el perfil de las familias. La experiencia regional. (LC/G.1761-P). Santiago de Chile. Recuperado el 10 de mayo de 2004 de http://www.eclac.cl/cgibin/getProd.asp?xml=/publicaciones/xml/5/5685/ P5685.xml\&x $=/$ mujer $/ \mathrm{tpl} / \mathrm{p} 9$ f.xsl\&base $=/ \mathrm{tpl} /$ top-bottom.xslt.

Comisión Económica para América Latina (CEPAL) (1994). Las mujeres en América Latina y el Caribe en los años noventa: elementos de diagnóstico y propuestas (LC/L.836(CRM.6/4)-P/E). Serie Mujer y Desarrollo, 18. Santiago de Chile. Recuperado el 10 de marzo de 2004 de 
http://www.eclac.cl/cgibin/getProd.asp?xml=/publicaciones/xml/5/5685/P5685.xml $\& \mathrm{x}=/ \mathrm{mujer} / \mathrm{tpl} / \mathrm{p} 9 \mathrm{f} . \mathrm{xsl} \&$ base $=/ \mathrm{tpl} /$ top-bottom.xslt.

Cuadrado, I., Navas, M. \& Molero, F. (2003). El liderazgo de hombres y mujeres: diferencias de género vs. diferencias de roles. Encuentros en Psicología Social, 2 (1), 243-248.

D'Elia, Y. (2004) La equidad. Recuperado el 16 de octubre de 2005, de http://www.vivavenezuela.nl/bestanden/ArticuloEquidadYDE.pdf

Dobles, I. \& Ruiz E. (1996). Violencia en la familia en Costa Rica: un estudio de opinión pública en población urbana. (Informe final de investigación). San José, Costa Rica: Centro Mujer y Familia/ Instituto de Investigaciones Psicológicas, UCR.

Friedan, B. (1997). Beyond gender. The new politics of work and gender. Washington, D.C.: The Woodrow Wilson Center Press.

Garrido, L. \& Palomero, C. (2003). La integración laboral de las mujeres jóvenes en suecia, España y Alemania. Encuentros en Psicología Social, 1 (4), 211-215.

Gil, E., Pujal, M., Ñañez, A.I., Laira, T. \& Martínez, L. (2003). Globalización y subjetividad: Una mirada de género. Encuentros en Psicología Social, 1 (4), 233-237.

Gólcher, R. (2000, 28 de octubre). Mujeres aún promueven machismo. La Nación, pp. 4A.

Gómez, M. (1996). Encuesta sobre satisfacción y valores básicos. (Informe de investigación). San José, Costa Rica: Proyecto Estado de la Nación.

Gónzalez, M. (1996). Jaque al rey: de la psicología patriarcal a la psicología feminista. Revista Costarricense de Psicologia, 12 (24), 10-30.

Hakim, C. (2005). Modelos de familia en las sociedades modernas. Ideales y realidades. Madrid, España: Centro de Investigaciones Sociológicas/Siglo XXI.

Herrera M. (1999, 21 de febrero). Pesimistas pero inconformes pero felices. La Nación, pp. $4 \mathrm{~A}$.

Inglehart, R. (1991). El cambio cultural en las sociedades industriales avanzadas. Madrid, España: CIS/Siglo XXI.

Instituto de Investigaciones Psicológicas (IIP) \& Organización Panamericana de la Salud (OPS).(1996). Algunas características psico-sociales de la juventud en la región metropolitana (Informe de investigación). San José, Costa Rica: Instituto de Investigaciones Psicológicas, Universidad de Costa Rica.

Limone, F. (2003). Una aproximación teórica a la comprensión del machismo. Encuentros en Psicologia Social, 1 (4), 220-223.

Lockwood, M. (1984). The women's movement and the family: A socio-historical analysis of constraits on social change. En Women and the family. Two decades of change. London: The Haworth Press.

Meentzen, A., \& Gomáriz, E. (2003). Introducción temática. En A. Meentzen \& E. Gomáriz (Eds.). Democracia de género. Una propuesta inclusiva. El Salvador: Fundación Heinrich Böll.

Méndez, W. (1999, 7 de febrero). Familia: meta de la juventud. La Nación, pp. 4A.

Méndez, W. (2000, 6 de junio). Plan contra agresión. Juzgados especiales verán casos de mujeres. La Nación, pp. 4A.

Pautassi, L. C., Faur, E., \& Gherardi, N. (2004). Legislación laboral en seis países latinoamericanos. Avances y omisiones para una mayor equidad (LC/L.2140-P/E). Serie Mujer y Desarrollo No. 56, Santiago de Chile. Recuperado el 10 de marzo de 2004 de http://www.eclac.cl/cgibin/getProd.asp?xml=/publicaciones/xml/2/15232/ P15232.x ml\&xsl=/mujer/tpl/p9f.xsl\&base=/tpl/top-bottom.xslt.

Preston, Ch. (2000). Social Continuity and Change, and Social Theory. Society \& Culture Association, 12th August. 2000. Recuperado el 10 de marzo de 2004 de http://www.google.com/search?q=cache:krlHTWiFPdcj:www.ptc.nsw.edu.au/scansw /docs/preston.doc+Social+Continuity+and + Change, + and + Social + Theory\&hl=es\&ct $=\mathrm{clnk} \& \mathrm{~cd}=1 \& \mathrm{gl}=\mathrm{cr} \& \mathrm{client}=$ firefox $-\mathrm{a}$. 
Schwartz, S.H. (2001) ¿Existen aspectos universales en la estructura y contenido de los valores humanos?. En V. Gouveia \& M, Ros (Eds.). Psicología social de los valores. Desarrollo teórico y metodológicos aplicados (pp. 53-77). Madrid, España: Biblioteca Nueva.

Szinovacz, M. (1984). Changing family roles and interactions. En B. Hess \& M. Sussman (Eds.). Women and the family. Two decades of change (pp. 163-201). London, United Kingdom: Haworth Press.

Vega, I. (1993). Cambio social, estructura y dinámica familiar en Costa Rica. Madrid: Editorial Universidad Complutense de Madrid. Colección Tesis Doctorales. No. 143/93.

Vega, I. (2000). Women in the microenterprise: Family organization and productive work. Family Science Review, 13, 3-4.

Vega, I. (2001). Las familias costarricenses en el contexto del nuevo milenio. En I. Vega \& A. Cordero (Eds.). Realidad familiar en Costa Rica: aportes y desafios desde las Ciencias Sociales (pp. 199-220). San José, Costa Rica: FLACSO-Costa Rica.

Vega R., I. (2003). Introducción. Relaciones de pareja y familia en la sociedad actual. En I. Vega (Ed). Pareja y familia en la sociedad actual: ¿Nuevos significados y desafíos? (pp. ix-xxi). San José: Instituto de Investigaciones Psicológicas. Universidad de Costa Rica..

Venegas, I. (2000, 14 de noviembre). Impulsan nuevo texto. La Nación, pp. 6A.

Vizcaíno, I. (1999, 6 de junio). Agresión doméstica. La Nación, pp. 4A.

Recibido: 21de julio de 2004 Aceptado: 14 de febrero de 2005 
